Cahiers de Narratologie

Analyse et théorie narratives

$20 \mid 2011$

Voix off et narration cinématographique

\title{
Le sens de l'écart : la narration déconstruite dans les vidéos de Willie Doherty
}

\section{Valérie Morisson}

\section{OpenEdition}

Journals

Édition électronique

URL : http://journals.openedition.org/narratologie/6355

DOI : 10.4000/narratologie.6355

ISSN : 1765-307X

Éditeur

LIRCES

Référence électronique

Valérie Morisson, "Le sens de l'écart : la narration déconstruite dans les vidéos de Willie Doherty », Cahiers de Narratologie [En ligne], 20 | 2011, mis en ligne le 26 août 2011, consulté le 19 avril 2019. URL : http://journals.openedition.org/narratologie/6355; DOI : 10.4000/narratologie.6355

Ce document a été généré automatiquement le 19 avril 2019

Article L.111-1 du Code de la propriété intellectuelle. 


\title{
Le sens de l'écart : la narration déconstruite dans les vidéos de Willie Doherty
}

\author{
Valérie Morisson
}

\section{NOTE DE L'AUTEUR}

Remerciements à Stacey Boldrick, Vincent Lavoie et René Monnier.

1 "Les choses ne sont jamais ce qu'elles semblent être ${ }^{1}$ ", entend-on dans l'une des vidéos de Willie Doherty, Somewhere Else, réalisée en 1998. Les choses ne sont jamais ce qu'elles semblent être, une phrase qui résume à elle seule le dessein de l'artiste. Ces choses sont avant tout des images et des mots, que ces derniers constituent un discours, un commentaire ou une parole. Le message transmis par l'artiste est éminemment politique. En effet, le travail du vidéaste qu'est Willie Doherty prend tout son sens lorsqu'il est perçu dans le contexte du conflit nord-irlandais. L'écart entre l'image et les mots, que Doherty ménage dans la plupart de ses œuvres, sert un propos : les décalages ourdis par l'artiste aboutissent à une remise en question de la criminalisation des militants et activistes, à une réflexion sur la victimisation, et à un réexamen de l'histoire nordirlandaise. L'œuvre suscite une confrontation pacifique entre deux points de vue qui, depuis la colonisation de l'Irlande et les premières plantations de l'Ulster à l'époque de Cromwell, apparaissent comme inconciliables. L'histoire contemporaine de l'Irlande du Nord hante à la fois les images et les textes conçus par Doherty. Né à London/Derry en 1959, il fut témoin de la mort de 13 militants lors du Dimanche Sanglant, le 30 janvier 1972. L'épisode s'avéra crucial pour l'artiste, qui rappela à plusieurs reprises à quel point cette tragédie fut formatrice. Le processus de paix en Ulster et les accords de Belfast, signés en 1998, ont rendu plus saillant l'impératif de réconciliation et ont ainsi mis à nu la 
nature profondément erratique du statut de victime, comme la difficulté de construire un récit objectif du conflit.

Les mots ont toujours été présents dans l'œuvre de Doherty. Ses premiers travaux photographiques incluaient des termes plaqués sur des paysages ou des visages de manière à pousser l'image dans ses retranchements et à dénoncer le parti-pris des médias. Dans ses installations vidéo, Doherty intègre non plus seulement des mots mais des récits, souvent déconstruits, où la voix off se révèle équivoque. Serge Daney affirmait que la voix-off était «stricto sensu, celle qui est toujours parallèle au défilé des images, qui ne les recoupe jamais » et ajoutait: "cette voix, surimposée après coup à l'image, montée sur elle, n'est susceptible que de métalangage. Elle ne s'adresse (tout entière, sa face énoncé et sa face énonciation) qu'au spectateur avec lequel elle fait alliance, contrat, sur le dos de l'image ${ }^{2}$ ». Dans beaucoup de productions cinématographiques, la voix off est en effet utilisée pour fournir au spectateur des éléments de compréhension que l'image ne donne pas ou que les personnages à l'écran ignorent. Dans les œuvres de Willie Doherty en revanche, le pacte unissant le spectateur à un acousmêtre omniscient est rompu. Si l'artiste simule une narration cinématographique c'est pour mieux déconstruire la perception audiovisuelle qui en est la caractéristique fondamentale. La voix off s'égare et divague, introduisant une discontinuité dans la narration et dans la focalisation. Le spectateur, actif dans sa quête d'une vérité insaisissable, est dès lors incapable de savoir qui parle à tel moment de la projection.

\section{Témoignages : entre introspection et rétrospection}

3 L'art vidéo, qui s'est développé après la mise au point des caméscopes portables à la fin des années 1960, soit après la naissance du cinéma parlant et de la télévision, s'est inévitablement constitué en rapport avec ces deux formes d'expression préexistantes. L'utilisation de la voix et de la narration par les artistes vidéastes s'est ainsi élaborée en opposition à d'autres récits audio-visuels, au point que la culture, la rhétorique et la sémiotique télévisuelles hantent l'art vidéo. Si les images filmées par les vidéastes n'ont que peu à voir avec les images télévisuelles, les modes narratifs s'en éloignent pareillement. A la différence du commentaire, transmis par une voix objective et publique, qui est l'usage dominant à la télévision, dans l'art vidéo, la narration prend volontiers une forme subjective, voire autobiographique, et relève davantage de la confession que du témoignage. Dans plusieurs installations vidéographiques, Willie Doherty rompt en effet avec les usages du reportage d'actualité et du documentaire dans la mesure où, d'une part, la voix off ne sert pas à commenter des images par le biais de descriptions, et où d'autre part, elle n'a pas d'effet vérité. La parole y est indirecte, qu'il s'agisse du souvenir tronqué d'un événement historique réactivé par des photos ou des actualités télévisuelles ou bien de récits transmis par des aïeux dont les souvenirs ont été altérés. Qui plus est, il n'y a pas un acousmêtre omniscient mais des voix plurielles désynchronisées; pas un récit historique mais des mémoires impures. Enfin, les installations évoquées ci-après, à l'exemple d'autres œuvres vidéo faisant éclater l'écran unique, sont fréquemment constituées de plusieurs larges écrans réunis en une seule pièce.

Dans de nombreuses œuvres, Willie Doherty altère le rapport entre l'image et la voix afin de dénoncer le manque de fiabilité des représentations médiatiques du conflit nordirlandais. "Le travail de Willie Doherty, explique Laurent Bossé, relecture critique de 
l'ensemble des modes de sémiotisation du terrorisme par médias interposés, s'élève contre certaines formes d'intrusion journalistique. Il suggère la difficulté de représenter et de donner à voir un état de conflit et, au-delà, toute situation de terreur, sans, précisément, l'alimenter et le prolonger ${ }^{3}$ ». A l'exemple d'autres artistes postmodernes, Willie Doherty, pour qui les œuvres de Warhol furent un modèle, puise dans la culture visuelle nord-irlandaise pour s'écarter des formes canoniques de représentation de la guerre.

5 C'est après avoir vu les images du Dimanche Sanglant diffusées par les médias, que Doherty s'avise que les représentations télévisuelles ne peuvent être que tronquées ${ }^{4}$. De manière plus générale, la couverture des Troubles et des actes terroristes par les medias britanniques fut pour le moins biaisée: «On transforme un événement précis, conjoncturel et politique, analyse Vincent Lavoie, en l'expression d'un conflit endémique, historique, insoluble ${ }^{5} »$. Doherty propose en contrepoint une réflexion sur les pouvoirs de l'image et des mots: "Je souhaitais utiliser ces informations recueillies de l'intérieur et tenter de travailler à partir d'images existantes (...) et j'ai pensé que si je pouvais trouver une position au sein du monde de l'art, ce serait plus intéressant pour faire éclore une forme de débat et permettre aux œuvres d'en faire partie ${ }^{6} »$. Jan Verwoert explique que la prolifération de la vidéo dans nos sociétés en fait un outil privilégié de la critique des formes culturelles et des modes de représentation, étant elle-même utilisée pour retravailler les formes médiatiques idéologiques de l'intérieur. Il n'est donc guère étonnant que Doherty se soit tourné vers cette forme devenue artistique. Comme d'autres vidéastes, il se trouve conséquemment à la fois consommateur-récepteur, auteur et critique des images télévisuelles, médiatiques et cinématographiques. La réappropriation des images d'archive dans certaines œuvres illustre cette position triple.

De l'aveu de plusieurs historiens, les images du conflit nord-irlandais ont été nombreuses, au point de hanter les consciences : "Répétées chaque semaine, les images de ce conflit se sont incrustées dans les esprits ${ }^{7} »$. Comme l'analyse Vincent Lavoie, chaque NordIrlandais reconnaît les «emblèmes d'un conflit qui, depuis le déploiement des troupes britanniques en 1969 , alimente la presse en imagerie psychodramatique ${ }^{8} »$. Dans les vidéos de Doherty, nombreux sont les personnages qui expliquent être obsédés par les images médiatiques du conflit, que ce soient des photographies de corps mutilés ou des images télévisuelles d'explosions. Dans $30^{\text {th }}$ January 1972 (1993), la voix off explique : « La tête de son compagnon explosa, comme dans un film ${ }^{9}$ ». Aucune image d'horreur, pourtant, n'accompagne ces mots; les paroles ne sont ni illustratives ni illustrées. Un narrateur dans Ghost Story (2007) s'avoue aussi possédé par les clichés médiatiques : «Je m'égarai dans les souvenirs de certains infimes détails des photographies de gens ou d'endroits que je ne connaissais pas. Des hommes emmenés, bandeau sur les yeux. Leurs mains fermement attachées par des liens en plastique. (...) Des corps empilés. Des vigils les surplombant et arborant un sourire pour les caméras. Des taches sur le sol blanc ${ }^{10}$ ». Pas plus que dans l'œuvre précédente, la confession n'est imagée ou la voix off incarnée. La vidéo montre alternativement les bords d'une route de campagne déserte et un parking où la silhouette sombre d'un homme apparaît brièvement. Le narrateur se rappelle également les images vacillantes d'un écran de télévision: "Je me souvenais d'images vacillantes que j'avais vues sur un écran de télévision ${ }^{11} »$. Le personnage de Tell Me What You Want (1996) affirme quant à lui : "Comme tout le monde je lisais les articles de journaux et l'avais vu à la télé1 ${ }^{12} »$. Un rapport officiel sur les victimes des Troubles affirme que toutes ont été exposées inlassablement aux images médiatiques du conflit ${ }^{13}$. 
Niklas Luhmann constatait de manière plus générale que «tout ce que l'on sait de nos sociétés, ou du monde dans lequel nous vivons, nous le savons par le biais des médias de masse $^{14}$ ». Critique, la voix off dirigée par Doherty énonce un métalangage et traduit la position de l'artiste immergé dans la société du spectacle.

Doherty est particulièrement sensible à l'utilisation de la voix off dans le film de propagande. The Only Good One is a Dead One (1993) constitue une réponse à une série de films courts conçus par le Ministère de l'Intérieur et invitant la population à collaborer avec la police ou à communiquer toute information sur d'éventuelles actions terroristes. Doherty analyse ces montages comme une manipulation visant à distiller le sentiment que tous les téléspectateurs étaient des victimes et à aggraver les peurs : « Ils mettaient nos peurs en images ${ }^{15}$ ». Son œuvre est constituée de deux séquences, filmées comme par une caméra de surveillance, qui n'enregistrent aucune action et qui sont accompagnées d'un témoignage en voix off équivoque et décousu. A l'action en accéléré du clip de propagande et aux invectives, Doherty substitue une scène vidée de tout potentiel dramatique et une confession inintelligible.

Doherty déconstruit la rhétorique des médias en supprimant la concordance entre image et voix. Habituellement alliées dans le discours journalistique pour mieux manipuler les consciences, elles se désaccouplent. Dans les vidéos de Willie Doherty, la voix off dément les images et en trouble la perception. C'est donc l'écart qu'il faut sonder car, comme l'explique l'artiste, ses images sont « réellement mises en scène et construites de manière à sembler 'cinématographiques, mais en même temps, elles abordent l'espace et le temps de manière spécifique ${ }^{16} »$.

9 L'une des découvertes des artistes utilisant la vidéo fut celle de la question de la durée et du montage. Les premières œuvres vidéographiques se conçoivent avant tout comme un enregistrement direct de performances ou d'actes. Celles des générations suivantes, empruntant davantage de procédés techniques au cinéma, sont le résultat de montages. Doherty, qui s'est à plusieurs reprises entouré de l'aide du réalisateur Seamus McGarvey, propose ainsi fréquemment des séquences discontinues mêlant des temporalités hétérogènes.

10 Si les images transmises en direct d'un conflit font appel en premier lieu aux passions et aux émotions, les images rétrospectives de Doherty, prises à froid, lorsque le sang et les dépouilles ont disparu, cherchent au contraire à susciter un regard dépassionné et distancié sur l'histoire et sa construction. Elles se veulent donc des "anti-scoops", comme le suggère Vincent Lavoie ${ }^{17}$. Son travail représente "le refroidissement de l'histoire en tant que symptôme morbide du traitement médiatique de l'actualité historique $^{18}$ ». L'une des intentions avouées de l'artiste est de travailler sur la confusion entre la réalité et la fiction, l'histoire et la mémoire, deux thèmes centraux dans 30th January 1972. L'œuvre emprunte au documentaire et au reportage pour inciter le spectateur à méditer le processus mémoriel. L'installation comprend trois moniteurs : l'un montre les images d'archives télévisuelles d'une foule massée dans Glenfada Park, où eurent lieu les évènements du Dimanche Sanglant ; les deux autres montrent le parc en 1993. A ces images se superposent trois pistes son: les bruits retravaillés de la manifestation de 1972 et des extraits d'interviews menées par l'artiste en 1993. Lors de ces échanges, Doherty avait demandé aux passants, vingt ans après la tragédie, s'ils se souvenaient soit de l'événement vécu, soit des images médiatiques diffusées à l'époque. Ceux qui se remémoraient l'événement ne figurent pas dans l'image, contrairement aux témoins oculaires de faits rapportés à la télévision. L'œuvre juxtapose les 
enregistrements de reportages d'archive, dans lesquels les voix renforcent l'effet vérité de l'image, et les paroles intimes ou les remémorations subjectives des interviewés qui portent un regard à la fois introspectif et rétrospectif. En cela, l'artiste s'éloigne des pratiques prévalant dans le reportage d'actualité.

11 A partir des années 1960, alors que les caméras se font plus légères permettant une prise de son en direct, la parole contribue à l'effet vérité de l'image en mouvement. De nouvelles techniques de transmission se sont avérées « toujours plus promptes à nimber l'événement des attributs de la simultanéité ", comme le souligne Vincent Lavoie ${ }^{19}$. S'éloignant à dessein de cette concordance temporelle et de l'illusion de concomitance absolue entre image et son, Doherty propose un regard distancié et critique sur la construction de la mémoire. Dans l'œuvre, ni l'image ni la parole ne forment un ensemble qui viendrait établir une vérité historique. Refusant toute monstration qui donnerait une autorité durable à ce qui ne peut être qu'une re-présentation, Doherty s'attache à démontrer que tout reportage d'actualité effectué 'à chaud' n'est qu'une réalité partielle dont le sens et le souvenir s'altèrent, se métamorphosent avec le temps. La projection vidéo a permis à de nombreux artistes d'explorer la dyade audio-vision, analyse que Doherty entreprend dans un contexte politique donné. Comme le remarque Marc Mayer, les premières œuvres vidéo renonçaient à raconter une histoire si bien qu' «à travers la projection vidéo, les artistes ont revisité la caractéristique essentielle du medium, à savoir le fait qu'il fut conçu pour enregistrer des informations audio-visuelles en temps réel. (...) Par conséquent, l'un des aspects les plus intéressants des projections vidéo créées par les artistes est leur réflexion sur le temps ${ }^{20} »$. Dans 30th January 1972, l'artiste propose plus précisément une réflexion sur la manière dont la mémoire, en se construisant dans la durée, s'appuie sur des représentations médiatiques.

Le projet de Willie Doherty était sous-tendu par l'idée que le Dimanche Sanglant constitue ce que psychologues et sociologues nomment un traumatisme culturel ${ }^{21}$. Ron Eyerman rappelle que la médiation et la reconstruction imaginaire sont centrales dans l'établissement du traumatisme national ou culturel: "Le traumatisme national ou culturel (...) est aussi ancré dans une série d'événements, mais pas nécessairement dans une expérience directe de ces événements. De telles expériences sont généralement médiées par les journaux, la radio, la télévision, par exemple, ce qui implique une distance spatiale et temporelle entre l'événement et la manière dont il est vécu ${ }^{22}$ ». Médiée, la représentation qui génère le traumatisme s'avère aussi déformée. En mêlant plusieurs enregistrements originaux dans son œuvre, Doherty rappelle que des sons, bruits ou voix, peuvent être raccordés à des images de manière artificielle et les faire mentir. Ses œuvres interrogent les partis pris et les procédés de la propagande - adoptant un discours que François Niney dit «surplombant et omniscient». Elles se rapprochent ainsi du documentaire contemporain où «l'image devient lacunaire, le commentaire personnel, et l'écart entre l'un et l'autre ouvre l'espace du doute et d'un espoir conditionnel ${ }^{23}$ ».

13 L'artiste invite les spectateurs à réfléchir non pas uniquement à la mémoire collective et nationale, mais également à la mémoire individuelle. Dans une œuvre de 1999 intitulée True Nature, à travers plusieurs témoignages transmis en voix off, Doherty évoque l'interférence constante $d u$ réel et de l'imaginaire, l'entrecroisement de l'histoire familiale, altérée au fil des générations, et des souvenirs personnels. L'installation se compose de cinq écrans où se succèdent de façon aléatoire des paysages irlandais et des scènes d'intérieur, réunis en un même espace, et flanqués de moniteurs télés où 
apparaissent les narrateurs. L'artiste a tout d'abord interviewé douze habitants de Chicago d'origine irlandaise mais n'ayant jamais été en Irlande, puis leur a demandé de décrire ce qu'ils imaginaient être des paysages irlandais et d'expliquer comment ils s'étaient forgés une image du pays de leurs ancêtres. Il a ensuite demandé à des Irlandais d'associer ces descriptions à des lieux réels. Dans la bande sonore définitive, trois personnes fournissent non pas des commentaires coïncidant avec les images mais des fragments discontinus de confessions. En effet, les paroles lacunaires des interviewés sont ponctuées par les interventions d'un acteur irlandais qui rapporte, de manière anachronique, les impressions d'un personnage dont on devine qu'il s'apprête à immigrer. Si la méthode de l'interview et certaines séquences filmées chez les personnes interviewées rappellent l'impression de vérité donnée par les documentaires, en déconstruisant la cohérence diégétique et en brouillant les focalisations, Doherty entrelace le passé, imaginé de manière rétrospective par les descendants d'immigrés, et le présent. A nouveau, la concordance de la voix et de l'image est abolie. Les fragments de récits ne sont pas recollés de manière à évoquer une histoire, pas plus que les images ne sont montées pour imiter la trame d'une narration.

Chez Doherty, comme chez les nouveaux documentaristes, «à la logique de l'identité et de l'adéquation, de la preuve par l'image et des représentants catégoriques - si caractéristiques de la statique et de la statistique des mass médias audiovisuels s'opposent des découpages-montages de la réalité assumant une dynamique de la contradiction, une profondeur du temps, des usages de la métaphore, des dispositifs d'interlocution ${ }^{24} »$. A ces ressorts, déclinés sur un mode poétique, s'ajoute l'intérêt de Doherty pour les traumatismes causés par la guerre civile.

\section{L'anachronisme comme symptôme d'un traumatisme}

«Ce que nous appelons la réalité est un certain rapport entre les sensations et les souvenirs qui nous entourent simultanément, rapport que supprime une simple image cinématographique, laquelle s'éloigne par là d'autant plus du vrai qu'elle prétend se borner à lui ", écrivait Marcel Proust ${ }^{25}$. Dans les vidéos de Willie Doherty, la voix-off sert de pendant à la réalité de l'image filmique, une image qui se trouve submergée par le flot incontrôlé des sensations et des souvenirs qui, selon Proust, constituent la réalité. Le souvenir et la remémoration occupent en effet une place centrale dans l'œuvre de l'artiste. L'affleurement du passé convoqué par la voix, qui se trouve dès lors en tension avec l'image, évoque le traumatisme subi par la population nord-irlandaise et l'impossibilité qui en découle de construire un récit conventionnel.

Dans les œuvres vidéographiques de Doherty, les narrateurs, que l'on ne peut pas toujours identifier comme personnages, doutent de leurs souvenirs. Dans How it Was (2001), si une seule scène est filmée, trois récits différents s'entrecroisent et les narrateurs, jamais montrés à l'écran, ont une mémoire parcellaire ou altérée des choses : «Tout a changé. J'étais là mais j'ai des doutes ${ }^{26}$ ». Le mélange des temps au sein du récit est le symptôme d'une mémoire où l'ordonnancement chronologique est bouleversé : « La lumière me joue des tours à cette heure de la journée. Tout ça s'est passé il y a si longtemps, c'est comme un monde différent. Je ne m'attendais pas à le trouver dans le tiroir, mais je pensai que je devais regarder. Au moins regarder. Je ne pourrais pas revenir pour vérifier. Tout a changé. Comme si tout était couvert de poussière. Je n'étais pas pressé $^{27}$ ». Lorsque la voix-off énonce des souvenirs, le spectateur imagine que le lieu 
associé à des violences passées, tel une ruine, suscite la réémergence de l'angoisse chez le narrateur. Parce que la confrontation à la ruine, fusse-t-elle celle d'une guerre, induit une collision entre le présent et le passé, elle empêche toute articulation et toute mise en récit. Selon le philosophe Dylan Trigg, la ruine est totalement envahie par un passé qui ne peut pas être reconstruit par le biais d'une narration conventionnelle, si bien que toute immersion dans un site associé au traumatisme induit une rupture temporelle du récit ${ }^{28}$. L'écart entre l'image et le texte, entre le lieu filmé et le récit ou la confession dans les œuvres de Doherty, est à envisager comme une manifestation matérielle de cette brèche. Aussi, si la perception de l'espace au cinéma est construite par la conjonction de l'image et du son, il en va de même pour celle du temps : l'espace présent en image peut, dans le cas de la ruine, donner de la profondeur au temps évoqué par le verbe.

Le fait que des fragments de récits surgissent de manière désynchronisée dans plusieurs œuvres de Doherty peut s'interpréter comme le symptôme d'un stress post-traumatique. Le traumatisme est associé non pas à l'événement mais au souvenir de l'événement et implique ainsi une période de latence durant laquelle le passé et le présent sont entremêlés au travers de représentations imaginaires ${ }^{29}$. Dominick LaCapra affirme que, dans que le souvenir traumatique, «certaines positions du sujet peuvent devenir prépondérantes ou écrasantes, telles que celle de la victime ou de l'agresseur... dans lesquelles on se trouve possédé par le passé et où l'on a tendance à le répéter de manière compulsive comme s'il était pleinement présent ${ }^{30}$ ». En raison de la violence du conflit interethnique qui a secoué l'Irlande du Nord pendant plus de vingt ans, de l'imprévisibilité des actes terroristes, de la répression et de l'omniprésence des véhicules de l'armée britannique, et des traces encore visibles de la guerre civile, cette dernière a indéniablement généré un traumatisme individuel et collectif ${ }^{31}$. Dans Bear in Mind These Dead, Susan McKay affirme que «le passé refuse obstinément de s'éloigner, en dépit de l'acharnement à le bannir ${ }^{32}$ ». La directrice du Wave Trauma Center affirme, quant à elle, que « pour beaucoup de ceux qui ont été touchés par les Troubles, le passé n'est pas le passé, il fait partie du présent ${ }^{33}$ ».

18 Les séquences vidéos de Doherty sont accompagnées de voix off évoquant des images violentes obsédantes : le souvenir est verbalement mis en images, d'où l'abondance de termes visuels dans les textes. Le narrateur de The Only Good One is a Dead One emploie les expressions: "Je vois", «Je visualise ma chute», «Je me vois presque», «Je revois la même scène ${ }^{34}$ ». Dans Tell me What You Want, des détails de paysages apparaissent à l'écran, accompagnés par le bruit du vent et de la pluie, puis sont interrompus par des plans montrant des silhouettes noires comme on en voit dans des reportages où les interviewés restent anonymes. Un homme - toujours hors-champ - rongé par la peur, persuadé que quelqu'un le suit ou qu'il va découvrir un corps enfoui dans les taillis, confesse: «Je vois ces événements horribles se dérouler comme s'il s'agissait d'une séquence dans un film ${ }^{35}$ ». Le sociologue Ron Eyerman attribue un rôle central aux représentations, notamment médiatiques, qui construisent ce qu'il nomme le traumatisme culturel $^{36}$. Nul doute que les images violentes que Doherty évite de reproduire, mais que les voix off évoquent, suscitent de l'angoisse.

19 L'impossible incarnation de la voix, sa nature perpétuellement acousmatique, peuvent également suggérer l'impact du traumatisme sur la construction du sujet. Arthur Neal associe ainsi le traumatisme culturel (par opposition au traumatisme individuel) à une perte de l'identité et du sens ${ }^{37}$. Si la névrose traumatique a été associée à la perte de réalité, les névroses de guerre, depuis les analyses fondatrices de Sándor Ferenczi, ont été 
liées à la rupture de cohérence intérieure du sujet, à un éclatement de la personnalité et au clivage. Pour G. N. Fisher le clivage résulte de l'impossibilité d'oublier s'exprimant de manière conjointe au besoin d'amnésie. Le traumatisme génère ainsi de multiples dissociations.

Dans Ghost Story, une œuvre filmée par Seamus McGarvey, la voix de Stephen Rea, un acteur nord-irlandais à l'accent aisément identifiable, évoque des souvenirs confus que l'on peut associer au Dimanche Sanglant. Comme dans d'autres œuvres de Doherty, les images qui défilent - celles d'une route traversant une forêt, d'un parking sombre n'illustrent pas les mots. La voix rauque et basse suggère un monologue intérieur qui pourrait être celui d'un homme au volant de sa voiture et perdu dans ses souvenirs. Pourtant, les yeux que Doherty filme par intermittence, sont ceux d'une femme. Comment donner un corps, dès lors, à cette voix masculine dans la mesure où la caméra subjective n'est plus la garantie d'une focalisation interne?

21 La spécificité des vidéos de Doherty est que les voix hors-champ ne sont pas celles de personnages qui ne sont plus à l'écran : ils n'apparaissent jamais. Les porteurs de ces voix ne sont pas non plus évoqués par un personnage principal, qui n'est d'ailleurs pas davantage incarné. Elles ne restent donc que des voix, des voix «en errance», comme dirait Michel Chion $^{38}$. Les focalisations et les ocularisations semblent internes mais leur rapport à la diégèse est problématique. Ainsi, jamais dans les vidéos de Doherty, la présence d'une voix humaine ne "structure l'espace sonore qui la contient ${ }^{39}$ " car elle n'est associée ni à un seul corps ni à une seule conscience. Les personnages restent des acousmêtres et le mystère de leur identité et de leur statut (de victime ou d'oppresseur) demeure.

\section{Voix-off et focalisation}

Jean Fisher rappelle que, comme la Palestine, l'Irlande du Nord est un territoire hanté par des récits divergents qui entretiennent des divisions : la violence «naît de l'échec du discours politique : dans l'intolérance, l'aveuglement, dans la différence et l'indifférence à la justice - en bref, dans l'incapacité à voir et à écouter ${ }^{40}$ ». Dans ses premières œuvres photographiques, Doherty convoquait des mots chargés de haine et accusateurs pour conjurer la spirale de violence qu'identifie Jean Fisher. Ces mots étaient ceux convoyés par les médias qui alimentaient le conflit. Dans les œuvres vidéographiques de l'artiste, la parole intime se substitue au discours public et l'identification du spectateur est utilisée de manière à évoquer l'ambivalence du statut de victime.

Jean Paul Fargier affirmait que «si la vidéo emprunte à la télévision ses meilleures armes et sa philosophie, c'est au cinéma qu'elle doit ses meilleurs modes d'emploi et sa stratégie ${ }^{41}$ ». L'effet sur le spectateur des œuvres de Doherty repose sur la multiplicité et l'équivocité de points de vue subjectifs et sur les tensions entre focalisation et ocularisation. L'artiste construit ainsi des atmosphères meurtrières rappelant celle de nombreux films noirs sans jamais donner corps aux fantasmes des spectateurs ni à leurs interprétations. Cadrage, prise de son et choix narratifs concourent à perturber le processus d'identification.

24 Dans Ghost Story, Willie Doherty utilise un steadicam de manière à ce que le spectateur soit immergé dans l'histoire et suive le regard d'un inconnu parcourant un terrain accidenté, tout en scrutant le paysage, que ce soit à la recherche d'indices ou pour se cacher lui- 
même. Le point de vue est construit de manière spatiale (en raison du caractère perspectif très marqué de l'image) autant que narratologique. Si la pénombre, le paysage sombre et inquiétant, les mouvements de caméra suggèrent une histoire de meurtre, il est impossible de savoir qui, de la victime ou du meurtrier, on suit. Troublante aussi est la constante proximité de la voix malgré les changements de focalisation : il n'y a pas une voix que l'enregistrement signalerait comme étant distante et qui se substituerait à une voix proche. Aucun élément technique d'enregistrement du son ne permet de distinguer les personnages différents qui témoignent ou réagissent à un paysage qui déclenche le souvenir.

Dans The Only Good One, la voix-off, celle de Paul McLoone, est associée à deux images différentes et, étant plus courte que les séquences filmiques, se trouve doublement disjointe (sur l'un des moniteurs s'affiche la vue depuis l'intérieur d'une voiture qui roule la nuit; sur l'autre, la même vue depuis une voiture immobile). La présence de pronoms sans référent identifié, ainsi que les glissements de points de vue rompent la cohérence diégétique: "Je ne me souviens plus maintenant quand j'ai commencé à me sentir exposé... Une cible légitime. Ca fait des semaines que je l'épie maintenant. Il fait les mêmes choses tous les jours... Tristement prévisible j'imagine. Ce salaud le mérite bien ${ }^{42}$ ». Him peut faire référence tantôt à une future victime épiée depuis un véhicule, tantôt à un assassin caché dans une voiture, tantôt à une victime représentée dans le journal, ou à un frère également évoqué de manière elliptique. Dans Somewhere Else, où Doherty joue avec les conventions du photojournalisme et du reportage d'actualité, la bande son accompagnant quatre projections simultanées est un collage de commentaires et de récits. L'emploi de pronoms personnels sans référent et leur alternance ôte toute crédibilité aux récits :

La caméra fixe la route, focalisée sur un point quelque part au loin. Au bout de quelques minutes une voiture apparaît non loin d'un virage. Elle continue à avancer sur la route, s'approchant régulièrement de la caméra. Soudain, la voiture s'arrête et un homme inconnu sort de la voiture et jette un objet non-identifié dans le taillis au bord de la route. Il remonte dans sa voiture et repart. Il commence à le / la [it dans le texte original] perdre. Il s'efforce de garder prise sur les choses. Une prise sur la réalité. Son corps fut découvert dans un fossé mal-odorant plein de carcasses d'animaux de ferme en décomposition ${ }^{43}$

L'identification du référent du pronom dépend de l'identité du locuteur; or, celui-ci demeure insaisissable. En outre, chaque micro-récit est parasité par des commentaires liés à la réalisation des séquences mais déconnectées de ces dernières ${ }^{44}$. Comme le résume une phrase du script, il s'agit ici à nouveau d'une histoire de première main mais avec un retournement ou une déformation:»a straightforward story with a twist». La voix acousmatique peut, dans le contexte dans lequel Michel Chion l'analyse, être perçue comme celle d'un maître chanteur, d'un narrateur omniscient manipulant le spectateur. Ce n'est pas le cas dans les œuvres de Doherty où le spectateur est actif et s'efforce, souvent en vain, de reconstruire un récit.

Si, dans la plupart des œuvres, les paysages restent déserts, malgré la présence proche d'une voix qui reste acousmatique, l'accent du locuteur donne cependant au spectateur un indice sur l'identité du ou des narrateurs. Dans They're All the Same (1991), une voix aux intonations irlandaises accompagne le portrait journalistique d'un homme au regard baissé. Les paroles sont syncopées : des descriptions de paysages irlandais sont ponctuées de phrases auto-accusatrices. 
L'air propre et doux n'est envahi que par des effluves langoureuses de tourbe. Je suis pathétique. Les talus verdoyants des chemins tortueux débordent de fuschias rouge sang. Je suis barbare. Nul part l'herbe n'est si verte et luxuriante. Je suis décent et honnête. C'est dans mes os. Nulle part le violet et le bleu des montagnes ne se parent de teintes si délicates. Je suis impitoyable et cruel ${ }^{45}$. uteur d'homme, tourne inlassablement autour d'un personnage qui correspond à l'image que l'on se fait d'un délinquant. La technique choisie permet l'absorption du spectateur, condition de l'efficacité de l'œuvre. Doherty s'est entouré de Seamus McGarvey pour la réalisation de cette séquence et a souhaité utiliser le visage connu de l'acteur irlandais Colin Stewart afin que les spectateurs croisent leur perception des personnages précédemment joués par l'acteur et celle de la vidéo. Stewart avait joué dans Holy Cross, un film pour la télévision réalisé par Mark Brozel et abordant les violences à Belfast. C'est à Kenneth Branagh que l'artiste a fait appel pour la lecture du script si bien que l'accent de la voix off est anglais et non nord-irlandais, une incongruité qui rappelle le doublage des interviews de militants irlandais par des Anglais lorsque le Broadcasting Act était en vigueur. Dans les années 1980, la voix de Gerry Adams fut doublée par un acteur en raison des interdictions imposées par l'Angleterre de diffuser la voix du membre du Sinn Fein. La BBC utilisait une voix-off ou bien des sous-titres lors des rares interviews d'Adams tandis que la RTE (Radio Telefis Eirann) prit la décision d'engager un acteur qui doubla parfaitement le leader du Sinn Fein. Dans Non-Specific Threat la discordance de la voix est donc politique. La scène, très sombre, se déroule en grande partie dans un entrepôt vide où une lumière blafarde éclaire le visage figé de l'homme observé. La voix peut être celle d'un policier ou d'un terroriste interrogeant un truand ou un traitre tandis que le cadrage rapproché et le regard panoptique permettent à l'artiste d'explorer les systèmes de représentation dans leur relation au pouvoir. La voix dit un texte non narratif qui recèle des contradictions et qui ne permet pas d'identifier le narrateur : «Je serai tout ce que tu voudras que je sois. Personne ne peut me connaître. Je suis tout ce que tu désires » (...) « Je suis le reflet de tes peurs. Je suis fictif. Je suis réel. (...) Je suis au-delà de la raison. Je t'ai contaminé. Je fais partie de ta vie. (...) Je fais partie de tes souvenirs » (...) «Je suis ta victime. Tu es ma victime. (...) Je partage tes peurs ${ }^{49}$ ». Doherty insiste sur l'impossible identification de la voix-off : « Non-Specific Threat aborde la manière dont on projette nos peurs et nos appréhensions sur un autre que l'on ne peut connaître ${ }^{50}$ ». Il ajoute: " notre seule expérience du meurtre est cinématographique ou télévisuelle (...). The Only Good One s'inspire des clichés cinématographiques de l'assassin

Cahiers de Narratologie, 20 | 2011 
et de la victime mais s'efforce de ne pas créer un personnage mais d'essayer de comprendre et d'explorer l'interdépendance de ces positions ${ }^{51}$ ».

Les problèmes lies à l'identification, voire la classification de la voix ramènent le spectateur à la question centrale dans l'œuvre de Doherty qu'est la reconnaissance des victimes. Le terme lui-même est ambigu puisque pour les travailleurs sociaux œuvrant à la réconciliation de populations en conflit, la victime peut être aussi l'auteur d'un délit. John D. Brewer analyse le dilemme identitaire des victimes qui « ont pendant longtemps défini leur identité par rapport à un ennemi et s'aperçoivent qu'au cours du processus de paix, elles doivent repenser leur perception d'elles-mêmes " ${ }^{52}$. Catholiques et Protestants, Irlandais et Anglo-Irlandais, Nationalistes et Unionistes rivalisent dans le processus de reconnaissance des victimes et ont des conceptions divergentes de la culpabilité ${ }^{53}$. Ce clivage politique et culturel est mis en scène dans les installations de Doherty. Dans The Only Good One is a Dead One, la même voix rapporte les idées qui hantent une victime et un assassin. Doherty lui-même explique que

le fait de vivre dans un pays en conflit vous oblige à envisager des réponses différentes à des circonstances difficiles : à comprendre les raisons qui poussent quelqu'un à agir violemment tout en en haïssant les conséquences; à admettre la peur que l'on a de devenir une victime de la violence tout en cherchant à se venger ; à percevoir un voisin, un ami, un membre de votre famille soit comme une victime, soit comme un agresseur ${ }^{54}$.

31 L'écart irréductible entre les voix et l'image est un moyen de rappeler qu'il est impossible d'établir une vérité, que les histoires se racontent de manières différentes, qu'il n'y a pas une victime mais des victimes. "Ce sur quoi l'artiste réfléchit, dit Carolyn Christov Bakargiev, est vrai de toutes les zones de guerre et de beaucoup de zones frontalières où les limites entre Soi et l'Autre semblent s'effriter, où les rôles de victimes et d'assassins se dissolvent et deviennent interchangeables, où la peur prend le dessus, où des conflits ethniques et les oppositions binaires (bien / mal, même / différent, colon / colonisé) remplacent la reconnaissance de la complexité des hommes et abolissent la conscience critique des structures du langage ${ }^{55} »$.

\section{Conclusion}

L'absence de synchronisation de la voix-off et de l'image et la déconstruction du récit dans les œuvres de Willie Doherty lui permettent d'attiser un regard critique sur les pouvoirs de l'audio-visuel, c'est-à-dire de l'effet vérité de l'image couplée au son; d'évoquer sur un mode poétique le traumatisme culturel ou national lié aux Troubles et au climat d'angoisse en Irlande du Nord ainsi que l'impossibilité de mettre en récit ces angoisses ; enfin, d'inciter les spectateurs à considérer l'équivocité du statut de victime et à se défaire d'un regard unilatéral sur le conflit. Pour ce faire, l'artiste emprunte à différents genres cinématographiques leurs techniques, leur rhétorique, leurs codes sémiotiques, sans jamais les parodier. L'inter-iconicité a des vertus critiques et non comiques, son métalangage étant le résultat non pas d'un positionnement esthétique mais de l'expérience directe du colonialisme. Carolyn Christov Bakargiev avance qu' « il y a toujours des résonances politiques dans l'œuvre de Doherty, mais même s'il parait s'inscrire dans la tradition d'un art radical et activiste au sens politique du terme, elle est en fait subtilement méta-politique. Plutôt que d'aborder les faits, elle aborde la manière dont on identifie ces faits et ce que l'on en fait sur un plan imaginaire ${ }^{56}$ ». L'œuvre de Doherty, dans laquelle la notion de diégèse est mise à mal, fait état de la nécessité de 
réécrire l'Histoire nationale dans un contexte où l'existence de la nation fait tant problème. Si comme l'affirme Brian Holmes, «l'art peut offrir à une société l'opportunité de réfléchir collectivement aux figures imaginaires sur lesquelles elle s'appuie pour concevoir sa propre cohérence et sa compréhension d'elle-même ${ }^{57}$ », le travail de Doherty en est une illustration.

\section{BIBLIOGRAPHIE}

\section{Catalogues d'exposition}

Willie Doherty : Unknown Depths, Cardiff, Derry, Glasgow, 1990, texte de Jean Fisher, "Seeing Beyond the Pale".

Willie Doherty, Musée d'Art Moderne de la ville de Paris, avril - juin 1996.

Willie Doherty: Same Old Story, London, Derry, Colchester, 1997, textes de Katherine Wood, Robin Klassnik et Liam KELLY.

Willie Doherty: Somewhere Else, accompagné d'un essai par I. Hunt, Tate Gallery, Liverpool, 1998.

Willie Doherty: Dark Stains, Donostia-San Sebastian, Koldo Mitxelena Kulturnea, 1999, accompagné d'un texte de Maité Lores, “The Streets Were Dark with Something More Than Night: Film Noir Elements in the Work of Willie Doherty".

Willie Dohery: How It Was, Belfast, Ormeau Gallery, Belfast, 2001.

Willie Doherty : False Memory, Merrell - Irish Museum of Modern Art, 2002, texts de Carolyn Christov-Bakargiev et Caoimhin Mac Giolla Léith.

Willie Doherty, Anthology of Time-based Works, Slide Installations and Videos, avec des textes de Yilmaz Dziewior, Matthias Mühling, et Francis McKee, Kunstverein in Hamburg, mai - septembre 2007, Städtische Galerie im Lenbachhaus und Kunstbau, Munich, septembre 2007 - janvier 2008.

Willie Doherty, Buried, Fruitmarket Gallery and Scottish Arts Council, Edinburgh, 2009.

\section{Ouvrages critiques}

BLAZWICK Iwona, « Willie Doherty », Art Monthly, n 172, décembre 1993 - janvier 1994, p. 4. BLOOMFIELD, Sir Kenneth, We Shall Remember Them, Report of the Northern Ireland Victims Commissioner, 1998.

BREWER, John D., « Memory, truth and victimhood in post-trauma societies », dans G. Delanty and K. Kumar, The Sage Handbook of Nations and Nationalism, Londres, Sage, 2006.

CARUTH, Cathy, Trauma : Explorations in Memory, Baltimore, Johns Hopkins University Press, 1995. CHION, Michel, La Voix au cinema, Cahiers du cinéma, Edition de l'Etoile, Essais, Paris, 1982, réédité en 1993. 
CORREIRA, Alice, «Crime and Justice : Willie Doherty and Chris Ofili », The Irish Review, no. 32, Thinking in Public (Automne - Hiver, 2004), Cork University Press, pp. 77-89.

DANEY, Serge, «L'Orgue et l'Aspirateur », Cahiers du Cinéma, nº 278-279, Août - Septembre 1977.

EYERMAN, Ron, Cultural Trauma: Slavery and the Formation of African American Identity, Cambridge University Press, 2001.

FARGIER, Jean-Paul, « Le Champ vidéo », Les Cahiers du cinéma, hors-série, n 10, octobre 1981.

HOLMES, Brian, « Artistic Autonomy », www.u-tangente.org

JOST, François, L'œil-caméra. Entre film et roman, Lyon, PUL, 1989.

Le récit cinématographique, Paris, Nathan, 1990.

LACAPRA, Dominick, History and Memory after Auschwitz, Cornell University Press, 1998.

LAVOIE, Vincent, « Image-évènement : représentations de la terreur dans l'œuvre de Willie Doherty et de Gerhard Richter », Parachute : Contemporary Art Magazine, Janvier, 1999.

MAUL, Tim, Interview with Tim Maul, Journal of Contemporary Art, vol. 7.2., New-York, 1995. Consultable à l'adresse suivante : http://www.jca-online.com/doherty.html

MAYER, Marc, Being \& Time, The Emergence of Video Projection, Albright-Knox Art Gallery, Buffalo, New York, 1996.

MOUFFE, Chantal « Artistic Activism and Agonistic Spaces », Art\&research : a Journal of Ideas, Contexts and Methods, Volume 1, n 2, été 2007.

NEAL, Arthur, National Trauma and Collective Memory, M. E. Sharpe Inc., 1998.

NINEY, François, L'Epreuve à l'écran, essai sur le principe de réalité documentaire, De Boeck Université, collection Arts et Cinéma, 2nd édition, 2001.

TRIGG, Dylan, « The Place of Trauma : Memory, Hauntings, and the Temporality of Ruins », Memory Studies, 2009, vol. 2.

\section{NOTES}

1. "Things are never what they seem ». Traduction de l'auteur.

2. Serge Daney, "L'Orgue et l'Aspirateur ", Cahiers du Cinéma, n 278-279, Août - Septembre 1977.

3. Laurent Bossé, Willie Doherty, Exposition 11 avril - 16 juin 1996, Musée d'Art Moderne de la ville de Paris.

4. «Je suppose que le meilleur exemple pour moi est le Dimanche Sanglant à Derry, où, âgé de 12 ans, j'ai vu de mes propres yeux treize personnes se faire tuer avant qu'on ne me dise que rien de cela n'était arrivé. Ce fut une expérience incroyablement décisive et ce fut important pour moi parce que j'ai pris conscience très clairement que toutes ces photographies étaient trompeuses. Mes photos abordent en grande partie l'idée que les gens se font d'une photo de l'Irlande du Nord. C'est cet écart entre ce qu'elles sont et ce qu'on pourrait espérer qu'elles soient qui m'intéresse et les montrer ici est pertinent parce que beaucoup de gens n'ont qu'une idée bizarre de ce qu'est l'Irlande, beaucoup n'y ont jamais été mais ont tout un tas d'images dans la tête ", Tim Maul, «Interview with Tim Maul », Journal of Contemporary Art, vol. 7.2., New-York, 1995. (Traduction de l'auteur).

5. Vincent Lavoie, «L'Image-événement: représentations de la terreur dans l'œuvre de Willie Doherty et de Gerhard Richter ", Parachute : essais choisis 1985-2000, vol. 2, Bruxelles, La Lettre 
volée, 2004, pp. 223-238, (Reprise de l'article paru en hiver 1999, n 93), p. 232. Voir à ce sujet Bill Rolston, The Media and Northern Ireland : Covering the Troubles, Londres, MacMillan, 1991.

6. Tim Maul, op. cit. (t.d.a).

7. Francis McKee, «Smithereens », dans Willie Doherty, Anthology of Time-based Works, Slide Installations and Videos, avec des textes de Yilmaz Dziewior, Matthias Mühling, et Francis McKee, Kunstverein in Hamburg, mai - septembre 2007, Städtische Galerie im Lenbachhaus und Kunstbau, Munich, septembre 2007-janvier 2008, p. 20.

8. Vincent Lavoie, op. cit., p. 234.

9. « His companion's head exploded just like you'd see it on a film » (t.d.a.).

10. (t.d.a.) «I became lost in memories of the minute details of photographs of people and places that I didn't know. Men being taken away blindfolded. Their hands tightly bound by plastic cables ties. (...) Bodies in a pile. Guards standing over them smiling for the camera. Stains on white floor ».

11. (t.d.a.) «I remembered things from a flickering television screen ».

12. (t.d.a.) « Like everyone else I read the newspaper reports and saw it on TV ».

13. Rapport publié par le Department of Health and Social Services of Northern Ireland, http:// www.ofmdfmni.gov.uk/livingwiththetraumaofthetroubles.pdf.

14. Niklas Luhmann, The Reality of the Mass Media, Cambridge and Oxford, 2000, p. 1.

15. Tim Maul, op. cit. (t.d.a).

16. Yilmaz Dziewior, "Conversation with Willie Doherty », dans Anthology of Time-Based Works, op. cit., p. 41. (t.d.a).

17. Vincent Lavoie, op. cit., p. 232.

18. Idem., p. 238.

19. Ibid.

20. Marc Mayer, Being \& Time, The Emergence of Video Projection, Albright-Knox Art Gallery, Buffalo, New York, 1996, p. 29.

21. Voir sur ce sujet Graham Dawson, «Trauma, Place and the Politics of Memory: Bloody Sunday, Derry, 1972-2004 ", History Workshop Journal - n 59, Printemps 2005, pp. 151-178 et Brian Conway, Commemoration and Bloody Sunday, Palgrave Macmillan, Memory Studies, 2010.

22. Ron Eyerman, Cultural Trauma: Slavery and the Formation of African American Identity, Cambridge University Press, 2001, p. 2.

23. François Niney, L'Epreuve à l'écran, essai sur le principe de réalité documentaire, De Boeck Université, collection Arts et Cinéma, 2nd édition, 2001, p. 9.

24. François Niney, op. cit., p. 11.

25. Marcel Proust, Le Temps retrouvé, cité par Niney, op. cit., p. 11.

26. « Everything is changed. I was there and I have doubts » (t.d.a.).

27. " The light plays tricks at that time of the day. It all happened so long ago, it's like a different world. I didn't expect to find it in the drawer, but I thought I should look. At least look. There was no going back to check. Everything is changed. As if everything was covered in dust. I wasn't in a hurry » (t.d.a.).

28. Dylan Trigg, "The Place of Trauma : Memory, Hauntings, and the Temporality of Ruins ", Memory Studies, 2009, 2, p. 87.

29. Cathy Caruth, Trauma : Explorations in Memory, Baltimore, Johns Hopkins University Press, 1995 , p. 17.

30. Dominick LaCapra, History and Memory after Auschwitz, Cornell University Press, 1998, p. 14.

31. Sir Kenneth Bloomfield, We Shall Remember Them, Report of the Northern Ireland Victims Commissioner, 1998, 2.10.

32. Cité par Fiona Bradley, "Uncommon ground", dans Willie Doherty, Buried, Fruitmarket Gallery and Scottish Arts Council, Edinburgh, 2009, p. 7. (t.d.a).

33. UTV website, http://www.u.tv/News/Troubles-trauma-victims-up-by-third, Tuesday, 04 May 2010. (t.d.a).

34. "I saw », «I visualize myself falling », "I can almost see myself », or « I see the same scene » (t.d.a.). 
35. "I see these horrific events unfold like a scene from a movie » (t.d.a.).

36. Roy Eyerman, op. cit., p. 3.

37. Arthur Neal, National Trauma and Collective Memory, M. E. Sharpe Inc., 1998, p. 31.

38. Michel Chion, La Voix au cinéma, Editions de l'Etoile/Cahiers du Cinéma, coll. (Essais), Paris, 1982 , p. 15.

39. Ibid.

40. Jean Fisher, «Seeing Beyond the Pale ", dans Willie Doherty: Unknown Depths, Cardiff, Derry, Glasgow, 1990, non paginé.

41. Jean-Paul Fargier, « Le Champ vidéo », Les Cahiers du cinéma, hors-série, $\mathrm{n}^{\circ}$ 10, octobre 1981.

42. "I don't remember now when I started feeling conspicuous ... a legitimate target. I've been watching him for weeks now. He does the same things every day... Sadly predictable I suppose. The fucker deserves it $»$ (t.d.a.).

43. «The camera looks straight up the road, focused on a point somewhere in the distance. After a few minutes a car appears around a bend in the road. It continues to drive along the road, steadily getting closer and closer to the camera. Unexpectedly, the car stops and an unknown man gets out of the car and drops an unidentified object into the hedgerow at the side of the road. He gets back into the car and drives off. He's staring to lose it. He struggles to keep a hold on things. A grip on reality » (t.d.a.).

44. "Fade up to a scene in a forest ", ou " Fade up to a wide shot of a disused industrial landscape ".

45. "The clean sweet air is interrupted only by the lingering aroma of turf smoke. I'm pathetic./ The verdoyant borders of twisting lanes are splattered with blood-red fuschia. I'm barbaric./ Nowhere is the grass so green or so lush./I'm decent and truthful. It is in my bones./ Nowhere are the purples and blues of the mountains so delicately tinged./ I'm ruthless and cruel » (t.d.a.).

46. Chantal Mouffe, «Artistic Activism and Agonistic Spaces», Art\&research : a Journal of Ideas, Contexts and Methods, Volume 1, n² 2, Eté 2007.

47. Voir Ed Moloney, "Closing Down the Airwaves : the Story of the Broadcasting Ban », dans Rolston, op. cit.

48. Mark Mayer, op. cit., p. 36.

49. "I will be anything you want me to be / I am unknowable / I am everything that you desire "(...) « I am the reflection of all your fears. I am fictional. I am real. (...) I am beyond reason. I have contaminated you. I am part of your life. (...)I am part of your memories. » (...)« I am your victim. You are my victim. (...) I share your fears » (t.d.a.).

50. Interview with Dziewior, op. cit., p. 41. (t.d.a.)

51. Interview with Tim Maul, op. cit.(t.d.a.)

52. John D. Brewer, « Memory, truth and victimhood in post-trauma societies », dans G. Delanty and K. Kumar, The Sage Handbook of Nations and Nationalism, Londres, Sage, 2006, p. 20. (t.d.a.)

53. Ibid.

54. Interview with Dziewior, op. cit., p. 42. (t.d.a.)

55. Carolyn Christov-BAKARGIEV, "Willie Doherty, In the Dark», Projected Works, Kunsthalle, Bern, 1996, p. 9. (t.d.a.)

56. Loc. cit., p. 16. (t.d.a.)

57. Brian Holmes, 'Artistic Autonomy and the communication society', Nettime, 26 Octobre 2003; conférence donnée à l'occasion de Diffusion: Collaborative Practice in Contemporary Art, Tate Modern, London. 


\section{RÉSUMÉS}

Dans les œuvres vidéographiques de l'artiste nord-irlandais Willie Doherty, les éléments narratifs entretiennent une relation complexe avec les images filmiques. Témoin de la guerre civile, récepteur méfiant des images du conflit, Doherty n'a de cesse de rappeler que la mémoire individuelle du traumatisme est erratique et le statut de victime problématique. La déconstruction du récit, l'instabilité de la focalisation, ainsi que l'écart, voire l'incompatibilité, entre l'image et le texte qui l'accompagne aboutissent à une réflexion sur les dissensions historiques. La voix off devient alors un facteur d'incertitude.

In his video works, all tightly focused on the Northern-Irish conflict, Willie Doherty probes the perpetrator-victim dyad and denounces the truncated visual representation of the conflict by deconstructing the relation between image and sound. The texts uttered by the voice-over raise political issues relevant to post-conflict Ireland, whether it be victimhood, national trauma or the construction of collective memory. Multiple open-ended interwoven narratives accompany images without closing their interpretation.

INDEX

Index chronologique : XXe siècle, XXIe siècle

Mots-clés : guerre civile, témoignage, traumatisme, victime, voix-off

Index géographique : Irlande du nord

\section{AUTEUR}

\section{VALÉRIE MORISSON}

Université de Bourgogne, Centre Interlangues, EA 4182 